\title{
An Improvement of the Kruk-Jaroniec-Sayari Method for Pore Size Analysis of Ordered Silicas with Cylindrical Mesopores
}

\author{
Mietek Jaroniec $^{*}{ }^{\dagger}$ and Leonid A. Solovyov ${ }^{\ddagger}$ \\ Department of Chemistry, Kent State University, Kent, Ohio, 44240 USA and Institute of Chemistry \\ and Chemical Technology, 660049 Krasnoyarsk, Russia
}

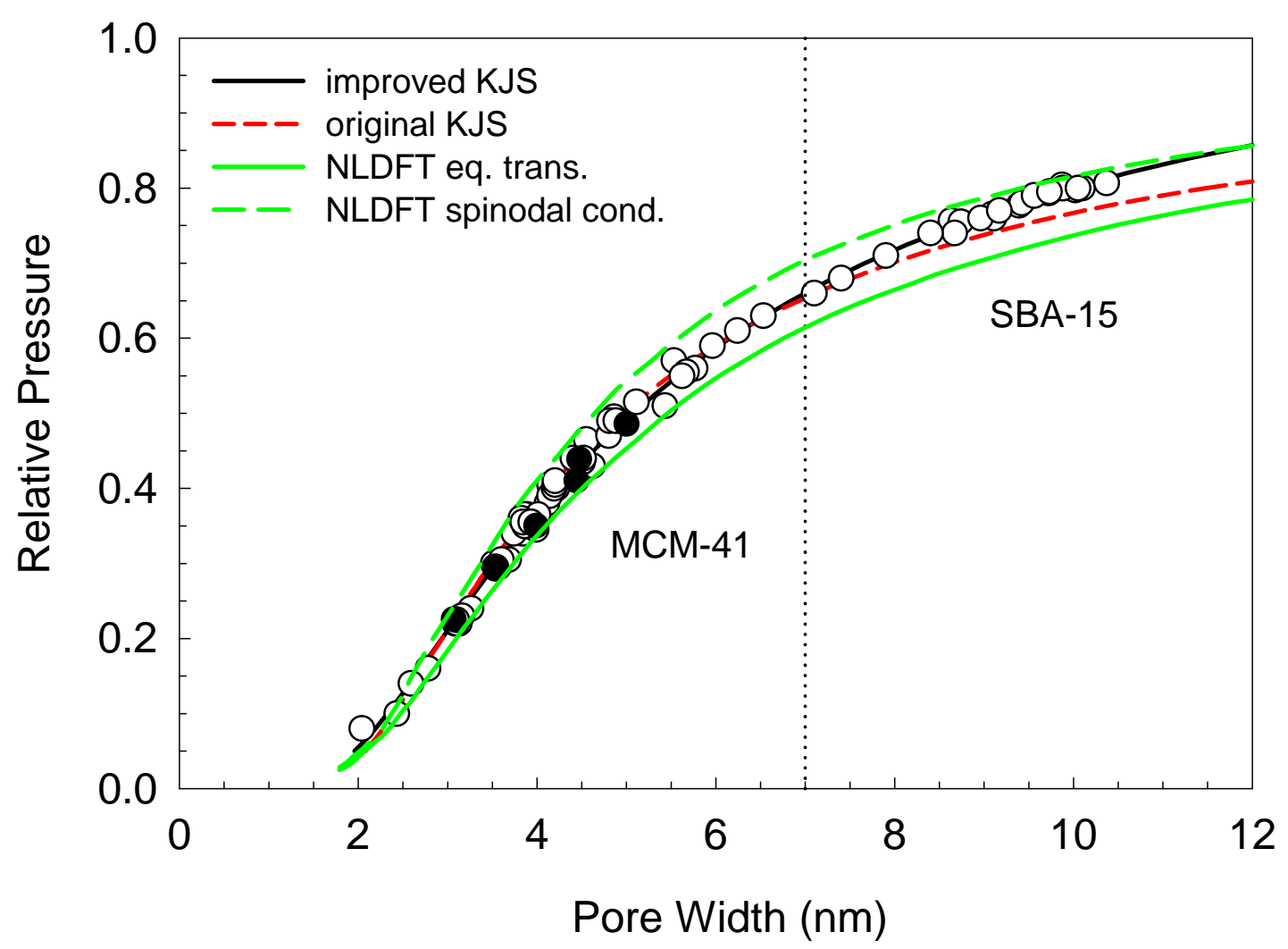

Figure S1. A comparison of the experimentally developed relation for the capillary condensation pressure versus the pore width with the KJS (Kruk-Jaroniec-Sayari) and NLDFT (Nonlocal Density Functional Theory) predictions. The black line represents the improved KJS dependence calculated according to eq 3 with $\mathrm{a}=1.15, \mathrm{~b}=0.875$ and $\mathrm{c}=0.27$, whereas the dashed red line represents the original KJS curve (eq 2), which was reported previously (Langmuir, 1997, 13, 6267). The dashed and solid green lines represent the NLDFT predictions for the spinodal condensation and equilibrium transition (Stud. Surf. Sci. Catal., 2000, 129, 597). The pore widths for the MCM-41 samples denoted by open circles, obtained by means of eq 1, were taken from Langmuir, 1997, 13, 6267; those denoted by filled circles were obtained by the XRD structure modeling using CDF-DDM method (Chem. Commun. 2006, 2242). The pore widths for the SBA-15 samples were obtained by the structure modeling using the SAXS data reported in J. Mater. Chem., 2005, 15, 5049.

\footnotetext{
*Corresponding author: Mietek Jaroniec (phone: 330-672 3790; e-mail: jaroniec@kent.edu).

${ }^{\dagger}$ Kent State University.

${ }^{\ddagger}$ Institute of Chemistry and Chemical Technology, Russia
} 
(C) American Chemical Society, Langmuir, la0609571, Supporting Info Page S2
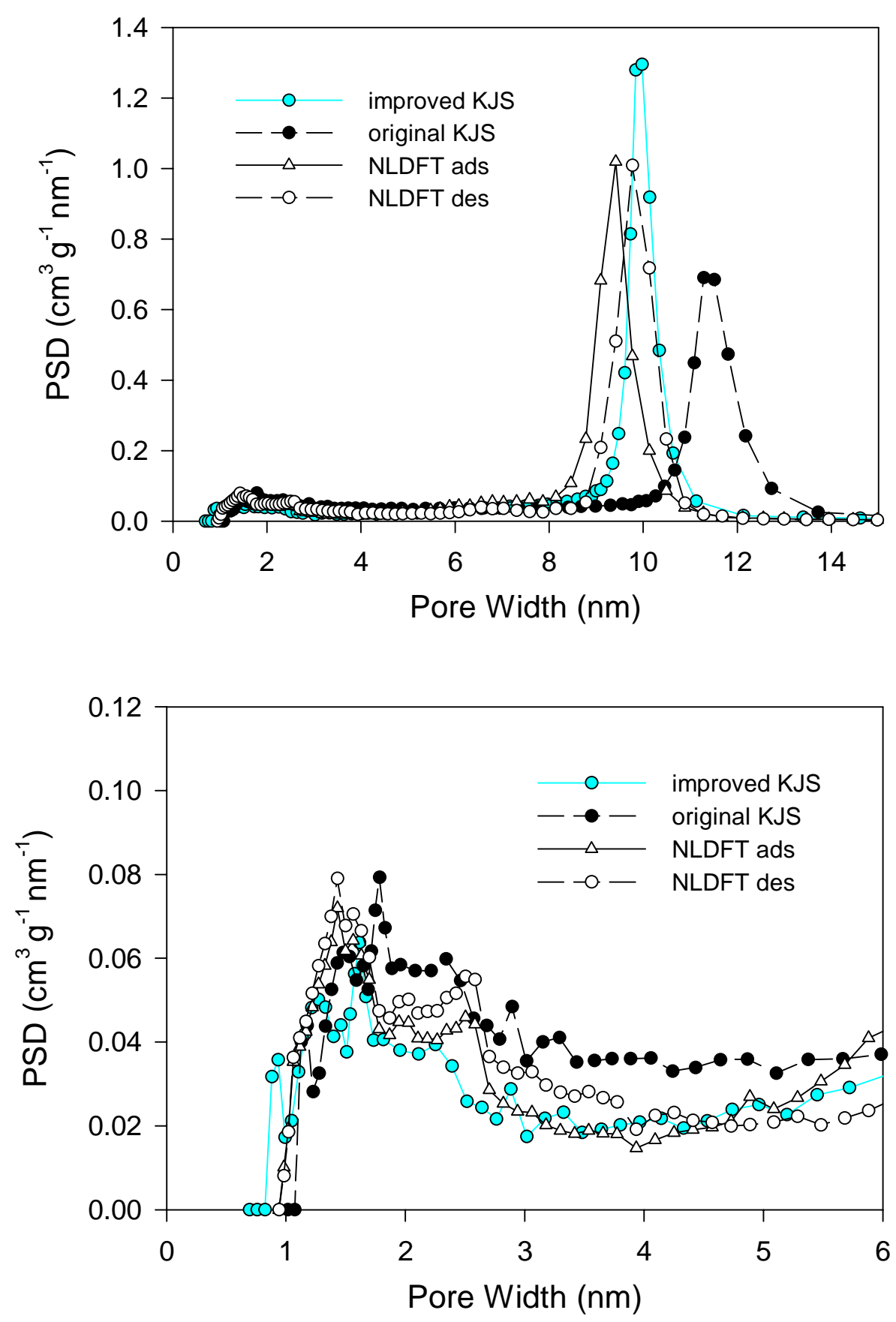

Figure S2. Pore size distributions (PSDs) calculated from adsorption branch of nitrogen adsorption isotherm for the SBA-15/72 sample by using original (black circles with solid line) and improved (blue circles with blue line) KJS methods in comparison to the PSD curves obtained by the NLDFT method using adsorption and desorption branches of the isotherm (open points with solid and dashed lines, respectively). 